\title{
Ocorrência de Pteroglossus bitorquatus Vigors, 1826 em Rolim de Moura, Rondônia: reflexões sobre uma espécie ameaçada de extinção
}

\author{
Occurrence of Pteroglossus bitorquatus Vigors, 1826 in Rolim de Moura, \\ Rondônia: reflections on an endangered species
}

\author{
Carlos Nei Ortuzar-Ferreira ${ }^{1 *}$, Alda d'Almeida Ortuzar Ferreira ${ }^{2}$, Wanderson Cleiton Schmidt- \\ Cavalheiro $^{3}$, Elvino Ferreira ${ }^{4}$
}

\begin{abstract}
RESUMO
As condições de fragilidade acarretando risco de extinção de espécies, principalmente em regiões antropisadas, é atualmente motivo de preocupação social. Este trabalho relata a ocorrência de uma espécie da família dos tucanos, o araçari-de-pescoço-vermelho (Pteroglossus bitorquatus), em perigo de extinção, registrado no município de Rolim de Moura, pertencente a Zona da Mata Rondoniense, a qual acumula os maiores índices de desmatamento do Estado e pertencente ao arco de desmatamento da Amazônia. Tal registro, discutido e pautado em revisão de literatura, aborda questões sobre as unidades de conservação bem como o papel do ecoturismo como oportunidade educativa e conscientizante. Serve como alerta sobre a necessidade do desenvolvimento de políticas públicas conservacionistas a fim de proteger a espécie em questão e seu ecossistema caso o quantitativo populacional não se encontrar tão reduzido a ponto da espécie entre em colapso nesta região.
\end{abstract}

Palavras-chave: Conservação; Amazônia; Aves; Tucano.

\begin{abstract}
The fragile conditions leading to the risk of species extinction, mainly in anthropized regions, is currently a matter of social concern. This work reports the occurrence of a species of the toucan family, the rednecked aracari (Pteroglossus bitorquatus), in danger of extinction, recorded in the municipality of Rolim de Moura, belonging to the Zona da Mata Rondoniense, which accumulates highest deforestation rates in the State and belonging to the Amazon deforestation arc. This record, discussed and based on a literature review, addresses issues about conservation units as well as the role of ecotourism as an educational and awareness-raising opportunity. It serves as an alert about the need to develop conservationist public policies in order to protect the species in question and its ecosystem if the population quantity is not so reduced to the point that the species collapses in this region.
\end{abstract}

Keywords: Conservation; Amazon; Birds; Toucan.

\footnotetext{
1 Universidade Federal Rural do Rio de Janeiro

*E-mail: carlosneiortuzarferreira@gmail.com

2 Universidade Federal de Goiás

3 Cavalheiro Engenharia Rural e Empresarial Ltda.

4 Universidade Federal de Rondônia
} 


\section{INTRODUÇÃO}

A conservação da biodiversidade mundial é, por vezes, o tema central dos debates atuais haja vista o cenário crítico em que muitos ecossistemas se encontram (FRANCO et al., 2015). Entretanto, conservar o que ainda nos resta é uma incumbência desafiadora que reúne esforços multigovernamentais envolvendo agências, instituições, organizações e outras esferas (FRANCO et al., 2015; PIGA; MANSANO, 2015). Para se ter uma ideia, a temática ambiental só entrou em voga a partir dos anos 1960, quando os problemas já eram considerados estarrecedores. Desde então, foram criadas cinco conferências internacionais para discutir a questão ambiental (e.g. níveis de emissão de poluentes na atmosfera, desmatamento e queimadas) com alguns acordos sendo firmados entre os países (e.g. Agenda 21, Protocolo de Kyoto) (PIGA; MANSANO, 2015). Além disso, pesquisadores do mundo todo têm voltado as atenções com preocupação para esse tema, desenvolvendo mecanismos de monitoramento do ambiente e dos seres vivos (SENA, 2018). Neste sentido, a União Internacional para Conservação da Natureza - UICN (em inglês International Union for Conservation of Nature - IUCN), criada em 1968, encarrega-se de fazer um levantamento geral acerca da biodiversidade de organismos existentes no planeta, bem como, estimar o grau de ameaça ou o risco de extinção para as espécies listadas (SENA, 2018; WIKIAVES, 2021).

Dentre os grupos animais existentes, as aves se destacam pela sua exuberância e diversidade, mas também por apresentarem inúmeras espécies ameaçadas ao longo do globo. Segundo a Lista Vermelha de Espécies Ameaçadas da UICN, aproximadamente, 40.000 espécies se encontram ameaçadas de extinção atualmente, sendo desse total, $13 \%$ aves (IUCN, 2021). Outro aspecto notável desse grupo é que, por responderem diferentemente aos distúrbios ambientais e apresentarem, de um modo geral, comportamentos conspícuos, as aves são consideradas excelentes bioindicadores (TEMPLE; WIENS, 1989). Assim, mensurar a riqueza e abundância do grupo de aves de uma determinada localidade é uma maneira segura para atestar a qualidade ambiental da área avaliada (PIRATELLI et al., 2008).

O araçari-de-pescoço-vermelho é uma ave da família dos tucanos (família Ramphastidae), de nome científico Pteroglossus bitorquatus Vigors, 1826 (PACHECO et al., 2021) e cujo status de conservação se encontra categorizado como "em perigo" (EN) na Lista Vermelha da UICN (Figura 1). A distribuição geográfica da espécie vai de 
leste a sul da Amazônia brasileira, incluindo registros para o território boliviano (eBIRD, 2022). Com isso em vista, nota-se uma marcante sobreposição da sua área de ocorrência com o arco do desmatamento (LEES; PERES, 2006; LEMOS; SILVA, 2011). Essa realidade traz ainda mais aflição quando constatada a carência de estudos sobre a biologia reprodutiva da espécie, por exemplo (WIKIAVES, 2020).

O presente trabalho tem por objetivo reportar a ocorrência dessa espécie no município de Rolim de Moura - RO, trazendo à tona a problemática do desmatamento histórico da região como um risco iminente para manutenção de populações da espécie na região, informando ainda, ao público, legisladores e cientistas a urgência de se adotar medidas conservacionistas não apenas na cidade, mas na Zona da Mata Rondoniense (ZMR) como um todo.

Figura 1. Status de conservação de Pteroglossus bitorquatus segundo a IUCN Red List.

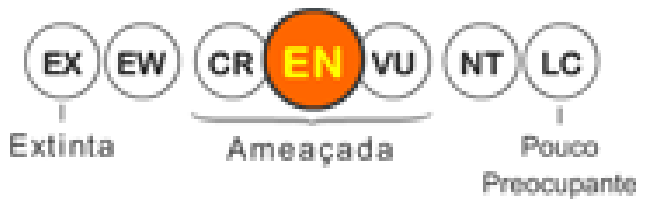

Fonte: WikiAves

No dia 13 de julho de 2014, em uma saída a campo para a prática de "birdwatching", foram observados cinco espécimes de Pteroglossus bitorquatus em um fragmento florestal na Linha 176 Norte, zona rural do município de Rolim de Moura, localizado na seguinte coordenada geográfica: $11^{\circ} 38^{\prime} 55.46^{\prime} \mathrm{S} 61^{\circ} 50^{\prime} 59.91^{\prime \prime} \mathrm{O}$. O fragmento em questão, situa-se a aproximadamente $8,5 \mathrm{~km}$ de distância, em linha reta, da malha urbana do município (Figura 2). 
Figura 2. Local do registro com sua referida distância em linha reta para a malha urbana do município.

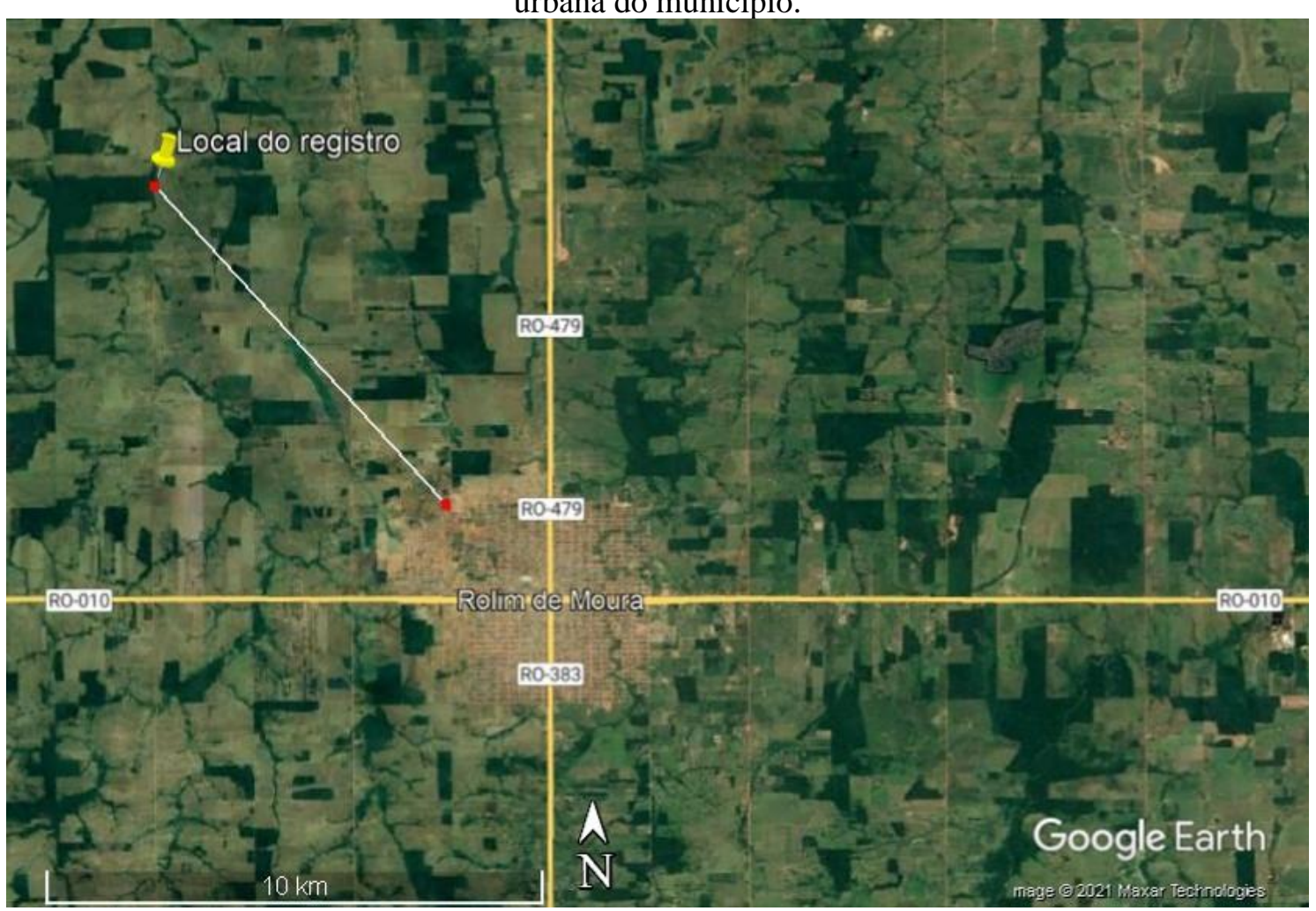

Fonte: Google Earth Pro

As identificações das aves foram realizadas mediante conhecimento prévio e corroboradas com as fontes de pesquisa: "Guia de Campo. Aves da Amazônia Brasileira" (SIGRIST, 2008) e site "Wiki Aves - A Enciclopédia das Aves" na página referente à espécie (WIKIAVES, 2020). Para fins de documentação comprobatória foram realizadas duas fotografias das aves (Figura 3) utilizando uma câmera Kodak easyshare max z990, lente 30xis wide angle, resolução de 12 megapixels, gravador Full HD 1080p. As fotos foram depositadas no banco de dados da plataforma digital de ciência cidadã "Wiki Aves - A Enciclopédia das Aves". A verificação do status de conservação da espécie foi feita na plataforma digital da IUCN Red List of Threatened Species (IUCN, 2016) disponível na internet, e confirmou que a espécie em questão é ameaçada de extinção. 
Figura 3. Registros fotográficos de Pteroglossus bitorquatus no município de Rolim de Moura. A. Indivíduo de Araçari-de-pescoço-vermelho (Pteroglossus bitorquatus) com um Pica-paubenedito-de-testa-vermelha (Melanerpes cruentatus) alçando voo na sua frente. B. Indivíduo sozinho.

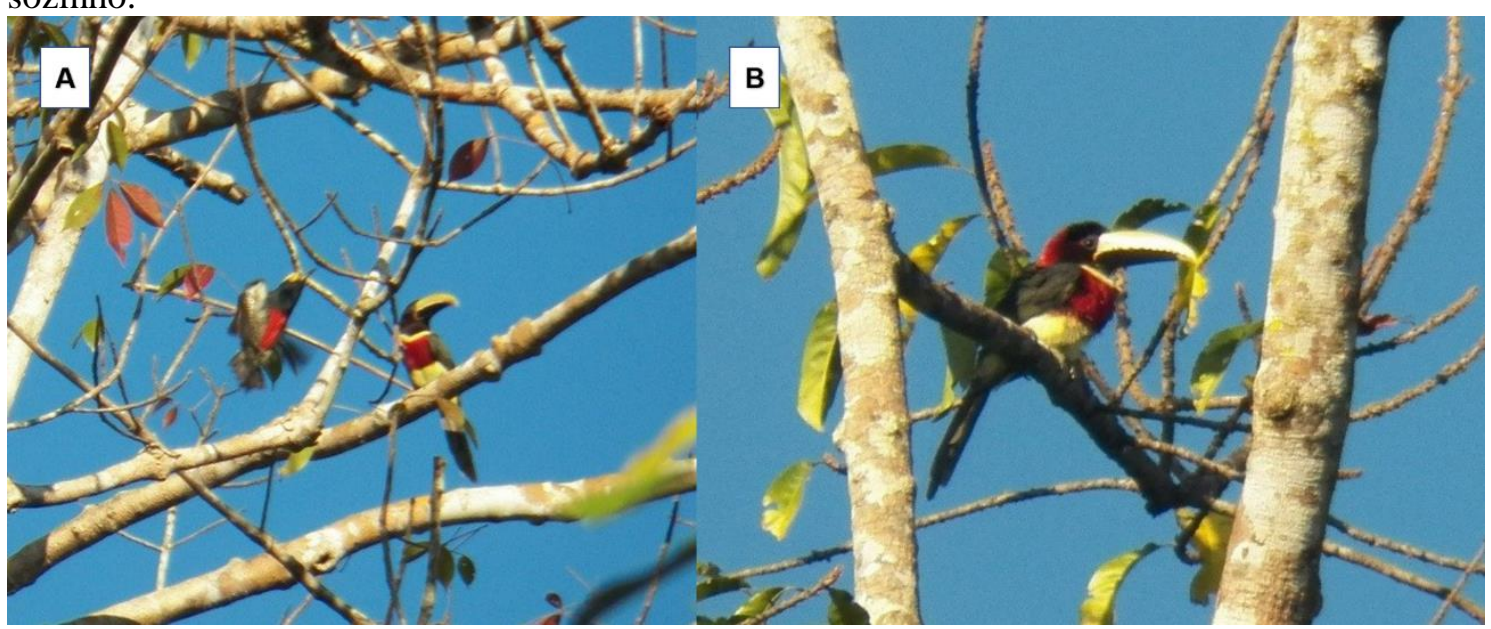

Fonte: O autor Carlos Nei Ortúzar Ferreira.

O fenômeno da extinção pode ser explicado de diferentes maneiras, mas em geral, quando de origem antropogênica, como na destruição de hábitats, são processos graduais que consistem em pequenas extinções locais até um determinado ponto onde não haja mais viabilidade populacional para uma espécie se manter (PURVIS et al., 2000). Entretanto, Lees e Peres (2006) apontam para um rápido colapso da avifauna no arco do desmatamento na Amazônia. Ainda nesse assunto, recentemente, muitos noticiários reportaram a extinção do Pica-pau-bico-de-marfim (Campephilus principalis) nos Estados Unidos, espécie célebre a qual muitos atribuem influência na criação do famoso desenho animado "Pica-Pau" (MENQ, 2021). Aqui no Brasil, também não faltam exemplos de espécies de aves que foram extintas da natureza: Anodorhynchus glaucus, Glaucidium mooreorum e Cichlocolaptes mazarbarnetti são algumas (PACHECO et al., 2021). Segundo levantamento feito por Lucena (2016), existem 41 espécies de aves consideradas ameaçadas na Amazônia, entre elas Pteroglossus bitorquatus. Ainda segundo a autora, o atual número de Unidades de Conservação (UCs) - categoria de Proteção Integral não se mostra suficiente para mantê-las em segurança, e expandir esse número nas áreas consideradas prioritárias para conservação seria uma solução inteligente para conciliar a diversidade biótica com os planos de desenvolvimento humano, mitigando assim, possíveis conflitos.

Atualmente, o estado de Rondônia conta com 80 Unidades de Conservação (entre partições públicas e privadas) de acordo com dados de Nagem (2021). Contudo, apesar 
de legalmente amparadas por lei, essas áreas protegidas não estão livres de apropriação indevida e crimes ambientais. Muitos são os casos de práticas ilegais por grileiros e madeireiros em Unidades de Conservação e terras indígenas, áreas que deveriam estar resguardadas (PEDLOWSKI et al., 1999; COSTA et al., 2015). Contumaz, o estado acumula recordes trágicos em relação aos níveis de desmatamento nas últimas décadas (PIONTEKOWSKI et al., 2014).

Esse problema ambiental no estado remonta às políticas desordenadas de colonização de suas terras (TRUBILIANO, 2016). Em especial, a Zona da Mata Rondoniense acumula um dos maiores índices de desmatamento do estado (SCHMIDTCAVALHEIRO et al., 2015; BORCHE, 2019). Em estudo conduzido por SchmidtCavalheiro et al. (2015) ficou demonstrado que a região da Zona da Mata Rondoniense acumulou um índice de 64,4\% de desmatamento acumulado, entre os anos de 1985 a 2011. O município de Rolim de Moura, liderou esse ranking negativo com assustadores 81,6\% (SCHMIDT-CAVALHEIRO et al., 2015). Dessa forma, o desmatamento na região é uma realidade crível que ameaça inúmeras espécies, como Pteroglossus bitorquatus. Indispensável ressaltar também que a Zona da Mata possui apenas cinco Unidades de Conservação nos seus $17.120,278 \mathrm{~km}^{2}$ de demarcação de área. São elas: Parque Estadual Ilha das Flores, Reserva Biológica Guaporé e Reserva Extrativista Pedras Negras em Alta Floresta D’Oeste e Parque Estadual Corumbiara e Reserva Particular do Patrimônio Natural Fazenda Bosco em Alto Alegre dos Parecis (Tabela 1). Rolim de Moura não possui nenhuma (ISA, 2014; SCHMIDT-CAVALHEIRO et al., 2015; WIKIPARQUES, 2018; ISA, 2022). 
Figura 3. Delimitação geográfica da Zona da Mata Rondoniense.
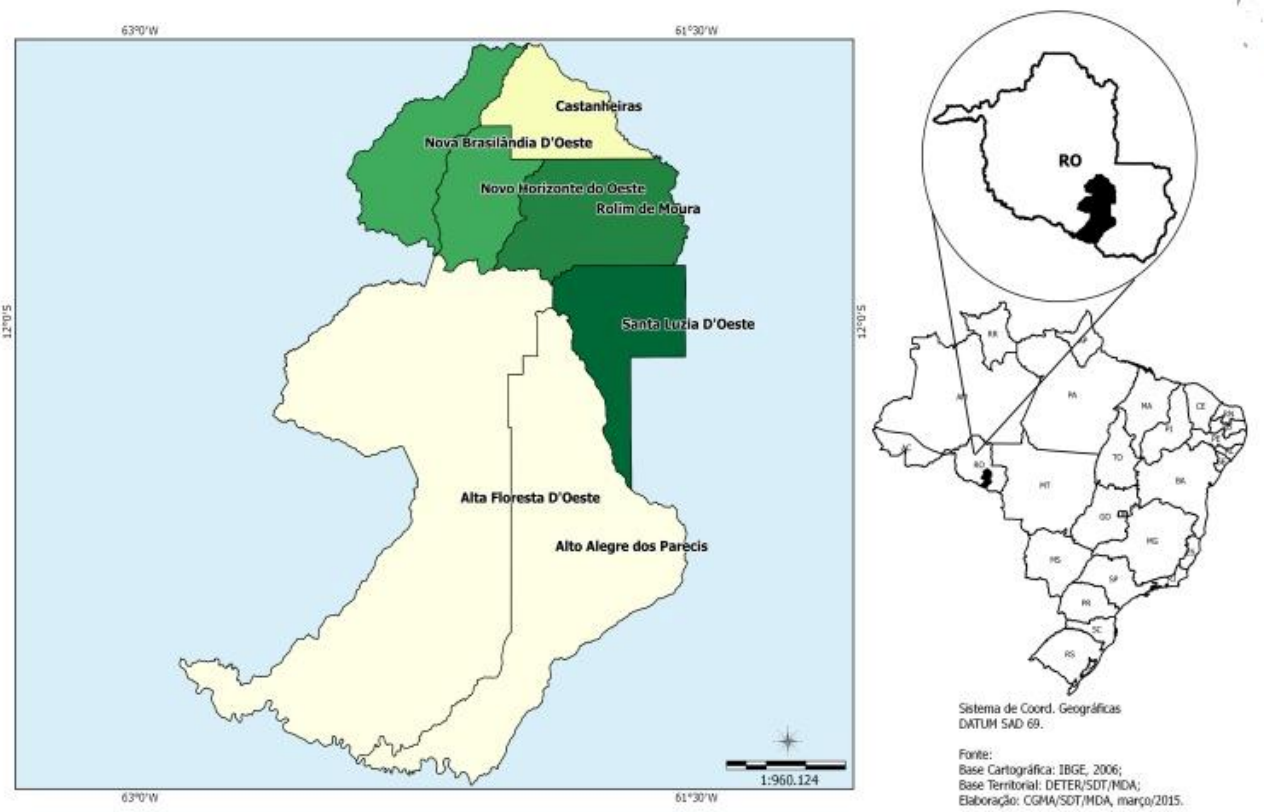

Fonte: http://sit.mda.gov.br/download/caderno/caderno_territorial_216_Zona\%20da\%20Mata\%20-

\%20RO.pdf

Tabela 1. Unidades de Conservação contidas nas delimitações da Zona da Mata Rondoniense

\begin{tabular}{llcc}
\hline Unidade de Conservação & \multicolumn{1}{c}{$\begin{array}{c}\text { Município } \\
\text { contemplado }\end{array}$} & $\begin{array}{c}\text { Extensão } \\
\text { territorial } \\
\text { da UC }\end{array}$ & $\begin{array}{c}\text { Percentual } \\
\text { contido na } \\
\text { ZMR }\end{array}$ \\
\hline $\begin{array}{l}\text { Reserva Biológica } \\
\text { do Guaporé }\end{array}$ & $\begin{array}{l}\text { Alta Floresta } \\
\text { D'Oeste }\end{array}$ & $6000,00 \mathrm{~km}^{2}$ & $15,56 \%$ \\
$\begin{array}{l}\text { Reserva Extrativista } \\
\text { Pedras Negras }\end{array}$ & $\begin{array}{l}\text { Alta Floresta } \\
\text { D'Oeste }\end{array}$ & $1244,09 \mathrm{~km}^{2}$ & $24,96 \%$ \\
$\begin{array}{l}\text { Parque Estadual } \\
\text { da llha das Flores }\end{array}$ & $\begin{array}{l}\text { Alta Floresta } \\
\text { D'Oeste }\end{array}$ & $899,77 \mathrm{~km}^{2}$ & $100 \%$ \\
$\begin{array}{l}\text { Parque Estadual } \\
\text { Corumbiara }\end{array}$ & $\begin{array}{l}\text { Alto Alegre } \\
\text { dos Parecis }\end{array}$ & $3840,55 \mathrm{~km}^{2}$ & $20,29 \%$ \\
$\begin{array}{l}\text { Reserva Particular do } \\
\begin{array}{l}\text { Patrimônio Natural Fazenda } \\
\text { Dom Bosco }\end{array}\end{array}$ & $\begin{array}{l}\text { Alto Alegre } \\
\text { dos Parecis }\end{array}$ & $486,72 \mathrm{~km}^{2}$ & $100 \%$ \\
\hline \hline
\end{tabular}

Fonte: Adaptado de Unidades de Conservação no Brasil (Disponível em:

https://uc.socioambiental.org/pt-br/arp/5559; https://uc.socioambiental.org/pt-br/arp/829;

https://sistemas.icmbio.gov.br/simrppn/publico/detalhe/654/; https://uc.socioambiental.org/ptbr/arp/1320; https://uc.socioambiental.org/pt-br/arp/1320; https://cidades.ibge.gov.br/brasil/ro), acesso em 20 de janeiro de 2022. 
Diante desses fatos, torna-se impreterível pensar na necessidade de criação de novas Unidades de Conservação na Zona da Mata Rondoniense, especialmente em Rolim de Moura, não deixando de recrudescer a fiscalização nas já existentes. Outra alternativa interessante seria fomentar na população, o anseio por práticas mais sustentáveis, mostrando-lhes o meio ambiente como um aliado e não como um fator limitante ao desenvolvimento (SPAOLONSE; MARTINS, 2016). O ecoturismo por exemplo, é uma prática que vem crescendo cada vez mais no Brasil e no mundo, sobretudo o "birdwatching", inclusive no ponto de vista financeiro (FARIAS, 2007; ALEXANDRINO et al., 2012). Segundo Alexandrino et al. (2012) a observação de aves possibilita, simultaneamente, educação ambiental e geração de renda. Em outro estudo, Anjos et al. (2021) apontam para a importância que remanescentes florestais de terras privadas exercem na manutenção de aves ameaçadas no bioma amazônico. Ou seja, a criação de Unidades de Conservação públicas ou mesmo RPPNs (Reserva Particular do Patrimônio Natural) ajudaria não só a conservar a biodiversidade, mas a movimentar a economia. Portanto, instigar à conscientização de práticas conservacionistas nas pessoas é sempre algo bem vindo.

\section{REFERÊNCIAS}

ALEXANDRINO, E. R., QUEIROZ, O. T. M. M. \& MASSARUTTO, R. C. O potencial do município de Piracicaba (SP) para o turismo de observação de aves (birdwatching). Revista Brasileira de Ecoturismo, v. 5, n. 1, p. 27-52, 2012.

ANJOS, L. J. S., PEREIRA, N. G. P., ALVES, S. T. M., JOSÉ, R. S. A. \& SOUZA, L. A. Forest remnants in private lands are critical to the persistence of endangered birds in an Amazonian hotspot. Journal for Nature Conservation, v. 61, 125984. doi: https://doi.org/10.1016/j.jnc.2021.125984, 2012.

BORCHE, M. I. A. F. S. Políticas que influenciaram na ocupação e desflorestamento na região da zona da mata rondoniense. Revista FAROL, v. 8, p. 303-313, 2019.

COSTA, G., SILVA, G., BRAMBILlA, C., LOBATO, L., CUNHA, L., TELES, V., NUNES, D., CALVANCANTE, M. Ocupações ilegais em unidades de conservação na Amazônia: o caso da Floresta Nacional do Bom Futuro no Estado de Rondônia/Brasil.

Revista de Geografia e Ordenamento do Território, v. 8, p. 33-49, 2015.

eBIRD. Red-necked Aracari: Pteroglossus bitorquatus. Recuperado de: https://ebird.org/species/renara1 - 2022 (acessado em: 10/01/2022).

FARIAS, G. B. A observação de aves como possibilidade ecoturística. Revista Brasileira de Ornitologia, v. 15, n. 3, p. 474-477, 2007. 
FRANCO, J. L. A., SCHITTINI, G. M., BRAZ, V. S. História da conservação da natureza e das áreas protegidas: panorama geral. Historiae, v. 6, n. 2, p. 233-270, 2015.

ISA. Instituto SocioAmbiental: Unidades de Conservação do Brasil - Rondônia, um pedaço da Amazônia - 2014, Recuperado de:

https://uc.socioambiental.org/noticia/142957 (acessado em: 13/01/2022).

ISA. Instituto SocioAmbiental: Unidades de Conservação do Brasil - 2022.

Recuperado de: https://uc.socioambiental.org/pt-br (acessado em: 13/01/2022).

IUCN. The IUCN Red List of Threatened Species: Pteroglossus bitorquatus - 2016. Recuperado de: https://www.iucnredlist.org/species/22728132/94971638 (acessado em: 10/01/2022)

IUCN. International Union for Conservation of Nature. The IUCN Red List of Threatened Species - 2021. Version 2021-3. Recuperado de:

https://www.iucnredlist.org (acessado em: 10/01/2022)

LEES, A. C., PERES, C. A. Rapid avifaunal collapse along the Amazonian deforestation frontier. Biological conservation, v. 133, p. 198-211. doi: 10.1016/j.biocon.2006.06.005, 2006.

LEMOS, A. L. F., SILVA, J. A. Desmatamento na Amazônia Legal: evolução, causa, monitoramento e possibilidades de mitigação através do Fundo Amazônia. Floresta e Ambiente, v. 18, n. 1, p. 98-108, 2011.

LUCENA, V. D. Áreas prioritárias para conservação na Amazônia - conservação das aves ameaçadas. 2016. 40 f. Dissertação (Gestão de Áreas Protegidas da Amazônia - GAP), Instituto Nacional de Pesquisas da Amazônia, Manaus, Brasil.

MENQ, W. Canal Planeta Aves - Pica-pau que foi extinto nos EUA (Pica-pau-bicode-marfim) e aves extintas no Brasil - 2021. Recuperado de:

https://www.youtube.com/watch?v=BIpL_lswwWY\&t=108s (acessado em: 10/01/2022).

NAGEM, J. T. F. K. Regularização fundiária de unidades de conservação: estudo de caso da Área de Proteção (APA) e da Floresta Estadual de Rendimento Sustentável (FERS) do Rio Pardo - Rondônia. 2021. 68 f. Trabalho de Conclusão de Curso, Universidade Federal Rural da Amazônia, Belém, Brasil.

PACHECO, J. F., SILVEIRA, L. F., ALEIXO, A., AGNE, C. E., BENCKE, G. A., BRAVO, G. A., BRITO, G. R. R., COHN-HAFT, M., MAURÍCIO, G. N., NAKA, L. N., OLMOS, F., POSSO, S. R., LEES, A. C., FIGUEIREDO, L. F. A., CARRANO, E., GUEDES, R. C., CESARI, E., FRANZ, I., SCHUNCK, F. \& PIACENTINI, V. Q. Annotated checklist of the birds of Brazil by the Brazilian Ornithological Records Committee - second edition. Ornithology Research, v. 29, p. 94-105, 2021.

PEDLOWSKI, M., DALE, V., MATRICARDI, E. A criação de áreas protegidas e os limites da conservação ambiental em Rondônia. Ambiente \& Sociedade, v. 2, n. 5, p. 93-107, 1999.

PIGA, T. R., MANSANO, S. R. V. Sustentabilidade ambiental e história: uma análise crítica. Revista Perspectivas Contemporâneas, v. 10, n. 2, p. 174-195, 2015. 
PIONTEKOWSKI, V. J., MATRICARDI, E. A. T., PEDLOWSKI, M. A., FERNANDES, L. C. Avaliação do desmatamento no estado de Rondônia entre $2001 \mathrm{e}$ 2011. Floresta e Ambiente, v. 21, n. 3, p. 297-306, 2014.

PIRATELLI, A., SOUSA, S. D., CORRÊA, J. S., ANDRADE, V. A., RIBEIRO, R. Y., AVELAR, L. H., OLIVEIRA, E. F. Searching for bioindicators of forest fragmentation: passerine birds in the Atlantic forest of southeastern Brazil. Brazilian Journal of Biology, v. 68, n. 2, p. 259-268, 2008.

PURVIS, A., JONES, K. E., MACE, G. M. Extinction. BioEssays, v. 22, n. 12, 1123 1133, 2000. doi: https://doi.org/10.1002/1521-1878(200012)22:12<1123::AIDBIES10>3.0.CO;2-C

SCHMIDT-CAVALHEIRO, W. C., VENDRUSCOLO, J., HILGERT-SANTOS, L. M., SANTOS, A. M. Impacto da colonização na Zona da Mata Rondoniense, Amazônia Ocidental-Brasil. Revista Geográfica Venezolana, v. 56, n. 1, p. 41-57, 2015.

SENA, N. K. Conservação da natureza em interface com a atuação da UICN (19472016). Tese (Doutorado em Desenvolvimento Sustentável), Universidade de Brasília, Brasília, 2018.

SIGRIST, T. Guia de Campo. Aves da Amazônia Brasileira. Avis Brasilis, São Paulo, 2008. 472 pp.

SPAOLONSE, E., MARTINS, S. S. O. Ecotursimo: uma ponte para o turismo sustentável. Revista Brasileira de Ecoturismo, v.9, n. 6, p. 684-698, 2016.

TEMPLE, S. A., WIENS, J. A. Bird populations and environmental changes: can birds be bio-indicators? American Birds, v. 43, n. 2, p. 260-270, 1989.

TRUBILIANO, C. A. B. Apontamentos sobre as frentes pioneiras na Zona da Mata Rondoniense (1970-2000). Albuquerque: Revista de História, v. 8, n. 16, p. 128-146, 2016.

WIKIAVES. WikiAves - A Enciclopédia das Aves: Araçari-de-pescoço-vermelho. (2020, nov. 10). Recuperado de: https://www.wikiaves.com.br/wiki/aracari-de-pescocovermelho (acessado em: 10/01/2022).

WIKIAVES. WikiAves - A Enciclopédia das Aves: Lista Vermelha IUCN. (2021, nov. 17). Recuperado de: https://www.wikiaves.com.br/wiki/lista_vermelha_iucn (acessado em: 10/01/2022).

WIKIPARQUES. Wikiparques: Estado de Rondônia cria 11 unidades de conservação (atualizado) (2018, mar. 27). Recuperado de:

https://www.wikiparques.org/noticias/estado-de-rondonia-cria-11-unidades-deconservacao/ (acessado em: 13/01/2022).

Recebido em: 03/02/2022

Aprovado em: 05/03/2022

Publicado em: 07/03/2022 